\title{
Study on Clinical Significance of LncRNA EGOT Expression in Colon Cancer and Its Effect on Autophagy of Colon Cancer Cells
}

This article was published in the following Dove Press journal: Cancer Management and Research

\author{
Yang Liu' \\ Bo Zhang' \\ Wen-Bin Cao' \\ Hai-Yan Wang ${ }^{2}$ \\ Lei $\mathrm{Niu}^{3}$ \\ Guo-Zhi Zhang' \\ 'Department of General Surgery, North \\ China University of Science and \\ Technology Affiliated Hospital, Tangshan \\ City, Hebei Province 063000, People's \\ Republic of China; ${ }^{2}$ Department of \\ Oncology, The 982 Hospital of the Joint \\ Logistics Support Force of the Chinese \\ People's Liberation Army, Tangshan City, \\ Hebei Province 063000, People's \\ Republic of China; ${ }^{3}$ Department of \\ Respiratory Medicine, Tangshan Hong $\mathrm{C}$ \\ Hospital Co. Ltd, Tangshan City, Hebei \\ Province 063000, People's Republic of \\ China
}

Background: Colon cancer (CC) is a common digestive tract tumor, and the increase of new and dead patients every year still puzzles clinical workers. LncRNA eosinophil granule ontogeny transcript (EGOT), as a newly discovered long-chain noncoding RNA (lncRNA), is differentially expressed in other tumors, but there are fewer studies of it in colon cancer.

Methods: The relative expression and diagnostic value of EGOT in CC were detected and analyzed by starBase online website and qRT-PCR. The patients were followed-up for five years, and Cox regression was used to analyze the independent prognostic factors of $\mathrm{CC}$. The effects of EGOT overexpression (pcDNA-RGOT) on CC cell function were detected by CCK-8, transwell and flow cytometry. WB was applied to detect autophagy. The influence of knocking out EGOT (sh-EGOT) on tumor growth was observed by tumor allogeneic inhibition. The microRNA (miR) and mRNA in the downstream of EGOT were predicted and the ceRNA network map was drawn.

Results: The online database and qRT-PCR detection showed that EGOT was highly expression in patients with $\mathrm{CC}$ and had good diagnostic value. The five-year survival rate of patients with high expression of EGOT decreased. EGOT and TNM staging were independent prognostic factors of patients with $\mathrm{CC}$. Functional analysis revealed that the growth and invasion abilities of cells increased, and the apoptosis rate decreased after overexpression. Upregulation of EGOT inhibited autophagy of CC cells and promoted cell growth. However, the tumor in nude mice was significantly lessened after knockout of EGOT. Bioinformatic analysis showed that microRNA-33a-5p and microRNA-33b-5p had targeted binding sites with EGOT.

Conclusion: EGOT is highly expressed in $\mathrm{CC}$ and has high diagnostic value. In addition, inhibition of EGOT can promote autophagy of $\mathrm{CC}$ cells and inhibit cell growth and metastasis, which is expected to be a potential therapeutic index.

Keywords: LncRNA EGOT, colon cancer, diagnosis, autophagy

\section{Introduction}

Colon cancer (CC) is still one of the general gastrointestinal tumors and one of the primary causes of carcinoma-related deaths. ${ }^{1}$ According to the report, ${ }^{2}$ it is estimated that more than 10 million new patients will be increased each year, and the number of dead patients will exceed 700 million. With the change of people's eating habits, the sick population tends to be younger in China. ${ }^{3}$ The prognosis depends on the stage of the disease at the time of diagnosis, with a five-year survival rate of $90 \%$ in early diagnosing and less than $10 \%$ in distal metastasis. ${ }^{4}$ However, patients with early diagnosed $\mathrm{CC}$ are not common in clinic, mainly
Correspondence: Guo-Zhi Zhang Department of General Surgery, North China University of Science and

Technology Affiliated Hospital, Tangsha City, Hebei Province 063000, People's

Republic of China

Tel +86-I583347I I00

Email myfypwly@I63.com 
because the patient's condition cannot be accurately detected by routine physical examination. ${ }^{5}$ Serum tumor markers CA199 and CEA have certain value in the diagnosis of CC, but their specificity is low. ${ }^{6}$ Therefore, it is urgent to develop rapid and highly sensitive $\mathrm{CC}$ screening markers to improve this situation.

In recent years, noncoding RNA (ncRNA) has gradually become a hot topic in various disciplines. ${ }^{7,8}$ According to its length, ncRNA can be divided into long-chain noncoding RNA (lncRNA) and microcoding RNA (microRNA). ${ }^{9}$ The research of microRNA in tumors has been reported greatly, but the study of lncRNA in tumors is still in the exploratory stage. The lncRNA is an RNA molecule with a length of more than 200 nucleotides. Early studies have shown that IncRNA cannot directly encode proteins, but they play a regulatory role in biological processes. ${ }^{10} \mathrm{~A}$ large number of studies have shown that IncRNA is closely bound up with the development and progression of $\mathrm{CC},{ }^{11,12}$ and it also plays a crucial role in the diagnosis and prognosis of CC. For example, studies by Zhou et $\mathrm{al}^{13}$ have shown that LncRNA XIRP2-AS1 predicted good prognosis of CC. Another study has shown that lncRNA CALIC upregulated $A X L$ to promote the metastasis of CC. ${ }^{14}$ LncRNA eosinophil granule ontogeny transcript (EGOT) is a newly discovered IncRNA, which is located on human 3p26.1 chromosome. $^{15}$ Early studies have shown that EGOT is highly expressed in hepatocellular carcinoma and breast cancer. ${ }^{16,17}$ Some studies have shown that EGOT can regulate autophagy of renal tubular cells induced by hypoxia. ${ }^{18}$ However, there is little research on whether EGOT affects the development and progression of $\mathrm{CC}$.

In this research, we have found that $E G O T$ is expressed to a high degree in cases of CC through TCGA database analysis, which suggests that EGOT may play a certain role in the progression of CC. Therefore, this research aimed to explore the clinical value and related mechanism of EGOT in CC, to provide potential therapeutic targets and diagnostic indicators for clinical practice.

\section{Materials and Methods GEPIA2 Online Analysis}

We logged into starBase ${ }^{19}$ (http://starbase.sysu.edu.cn) to choose the pan-Cancer $\rightarrow$ Gene Differential Expression, analyze the expression of EGOT in $\mathrm{CC}$ and draw a box diagram.

\section{Clinical Data}

From February 2012 to February 2014, 86 patients with CC who were treated in North China University of Science and Technology Affiliated Hospital were selected as the study group (SG). The serum of patients was collected before operation. The cancer tissues and adjacent tissues were collected during the operation. Then they were transported to the laboratory for detection by liquid nitrogen. In addition, the serum of 50 normal physical examinees who received medical examination in our hospital in the meantime were obtained and they were included in the control group (CG). There were no statistical differences in baseline data between the SG and CG. In this research, all patients were diagnosed with $\mathrm{CC}$ for the first time, and they had not received antitumour therapy before. All patients and physical examinees provided informed consent. This research was ratified by the Medical Ethics Committee of North China University of Science and Technology Affiliated Hospital, and conformed to the Declaration of Helsinki. ${ }^{20}$

\section{Cell Culture}

CC cells SW480, SW620, SW1116, LoVo, CaCo2 and normal colon mucosa cell line NCM460 were from American Type Culture Collection (ATCC; Manassas, VA, USA). The purchased cells were cultivated in DMEM containing (Gibco, CA, USA) 10\% FBS (Thermo Fisher Scientific, USA), and the cells were cultured in $5 \% \mathrm{CO}_{2}$ incubator at $37^{\circ} \mathrm{C}$.

\section{Cell Transfection}

To overexpress EGOT, the full-length EGOT sequence was introduced into cells by pcDNA3.1 plasmid (GenePharma, Shanghai, China). The constructed vector was named pcDNA-EGOT, and the blank pcDNA3.1 plasmid (pcDNA3.1-NC) was used as control. The cell transfection was conducted using lipofectamine 3000 reagent (Invitrogen, Carlsbad, CA, USA) according to the makers's protocol. After transfecting for $48 \mathrm{~h}$, the cells were collected for follow-up detection.

In addition, an EGOT knockdown (sh-EGOT) recombinant lentivirus and a control recombinant lentivirus (shNC) (Sangon Biotech Company, China) were constructed. The concentrated virus was used to infect $5 \times 105$ cells with $4-6 \mu \mathrm{g} / \mathrm{mL}$ in a six-well plate. Then infected cells were cultured with $1 \mu \mathrm{g} / \mathrm{mL}$ puromycin for two weeks. 
Stable knockdown cell strains were identified via qRTPCR.

\section{qRT-PCR}

Total RNA was extracted from the collected tumor tissues, serum and cells by Total agent. Then, the total RNA was reverse transcribed by the First Strand cDNA kit to obtain cDNA (Thermo Fisher Scientific, USA). The 7500 Fast Real-time PCR system (ABI, USA) and $\mathrm{SYBR}^{\circledR}$ PrimeScript $^{\mathrm{TM}}$ RT-PCR kit (Dalian, China) were applied for real-time PCR amplification. The amplification system and reaction conditions were configured according to the kit instructions. 2- $\triangle \triangle \mathrm{CT}$ was used to quantitative gene expression. ${ }^{21} G A P D H$ expression was used for normalization. EGOT upstream primer was 5'-GGTAGGTGGACT GCGTTGTT-3', and downstream primer was 5'-ATG GGGGAGCTGAGCACTTA-3'. GAPDH upstream primer was 5'-AGACTCGCTGATGATCCATGC-3', and downstream primer was 5'-AGGTGACCACAGTGTTCTG-3'.

\section{Detection of Cell Proliferation}

In this study, the cell counting kit-8 (CCK-8, Dojindo Molecular Technologies, Japan) was applied to detect cell growth. The transfected cells were resuspended to $5 \times 10^{3}$ cells/well, and then put in 96-well plate for culture. Then the CCK-8 solution $(10 \mu \mathrm{L})$ was put in each well at $0,24,48$, and $72 \mathrm{~h}$ respectively, and incubated for one hour. Absorbance analysis (A) was performed at 450 $\mathrm{nm}$ with a enzyme-labeling instrument (Bio-Rad, USA). The experiment was repeated three times.

\section{Detection of Cell Invasion}

Cells were collected and made into $1 \times 105$ cells $/ \mathrm{mL}$ cell suspension. The medium $(600 \mu \mathrm{L})$ comprising $10 \%$ FBS was put in the lower chamber, and the cell suspension (200 $\mu \mathrm{L}$ ) was put in the upper chamber (precoated Matrigel) and cultivated for $24 \mathrm{~h}$. Then the fluid from the upper and lower chambers was discarded. The cells in the lower chamber were fastened with $4 \%$ paraformaldehyde for 30 min. Thereafter, the paraformaldehyde was eliminated, and cells that did not pass through the film were wiped clean with cotton swabs. The lower chamber was dyed with $0.1 \%$ crystal violet for $10 \mathrm{~min}$, while the lower chamber was rinsed with PBS three times. Next, the migrated cells were analyzed and counted under a microscope (Olympus, Japan).

\section{Apoptosis Test}

Apoptosis was analyzed by flow cytometry. The cells in logarithmic growth phase were inoculated in a 96-well plate at a density of $1 \times 104$ cells/well, cultivated for $24 \mathrm{~h}$, rinsed twice with $\mathrm{PBS}$, fastened with $70 \%$ ethanol and conserved at $4{ }^{\circ} \mathrm{C}$ for one night. They were washed once with PBS to regulate the cellular density to $1 \times 10$ $6 \mathrm{mg} / \mathrm{mL}$. The final concentration was adjusted to $0.05 \mathrm{mg} / \mathrm{mL}$ by adding propidium iodinate staining solution, and the cells were dyed at $4^{\circ} \mathrm{C}$ for $30 \mathrm{~min}$. Then, the apoptosis was analyzed via flow cytometry (Miltenyi Biotec, USA). Each group was provided with three repeating wells.

\section{WB Test}

The transfected cells and tumor tissues of nude mice were collected and lysed in RIPA lysis buffer (Beyotime Biotechnology, Shanghai, China) to obtain total proteins. Then, $\mathrm{BCA}^{\mathrm{TM}}$ protein detection kit (Pierce, Appleton, WI, USA) was used to detect the concentration of protein extract. Then, it was isolated by $10 \%$ SDS-PAGE, and then moved to polyvinylidene fluoride (PVDF) film. The membrane was cultivated with $5 \%$ nonfat dried milk at ambient temperature for one hour, and Bcl-2, Bax, pro-caspase-3, cleavedcaspase-3, LC3- I , LC3- II, Beclin-1, p62 and primary antibody were added to incubate overnight at $4^{\circ} \mathrm{C}$. Then, the appropriate secondary antibody labeled with HRP (1:500, Abcam) was added at room temperature and incubated for one hour. After rinsing, chemiluminescence testing was performed. Anti- $\beta$-actin antibody (1:1000, Affinity, USA) was applied as intraprotein control.

\section{Immunofluorescence Staining}

Immunofluorescence staining was used to detect the changes of autophagy-related proteins in cells. After washing with PBS three times (five minutes each time), the cells were fixed for $10 \mathrm{~min}$, and then soaked with Triton X-100 (0.1\%) PBS solution for $10 \mathrm{~min}$. Then they were sealed at room temperature for one hour and incubated with anti-LC3A/B antibody at $4^{\circ} \mathrm{C}$ overnight. The next day, the cells were washed thoroughly with PBS and cultured at room temperature for three hours with Cy3-labeled goat anti-rabbit $\mathrm{IgG}$ (Beyotime, China). The chromatin was stained with DAPI for $10 \mathrm{~min}$, and the cells were evaluated under 
a fluorescence microscope (Olympus IX71, Olympus, Japan). ImageJ was used to quantify the fluorescence intensity of Alexa Fluor 488.

\section{Bioinformatics Analysis}

The potential microRNA of EGOT was predicted through online prediction websites of microRNADB, microRNA code, starBase, LncBase. For the predicted common microRNA, TargetScan, starBase, microRNADB and microRNADIP were applied to predict the potential common targeted genes of common microRNA. Gene Ontology (GO) and Kyoto Encyclopedia of Genes and Genomes (KEGG) were applied to analyze the targeted gene with R language "clusterProfiler" package, and the bubble diagram was drawn.

\section{In vivo Study}

The study was approved by the Medical Ethics Committee of North China University of Science and Technology Affiliated Hospital, and the animal experiment was conducted according to the Laboratory animal-guideline for ethical review of animal welfare issued by China in $2018 .^{22}$ Six four-week-old male BALB/c athymic nude mice (Viton Lihua, Beijing, China) were used for xenotransplantation experiment. SW620 cells infected with shEGOT and sh-NC $\left(2 \times 10^{7} / \mathrm{mL}\right)$ were digested by trypsin, then centrifuged, washed with PBS three times, and then resuspended in PBS. The cell suspensions were inoculated to the right side (sh-EGOT) of nude mice in sh-EGOT group $(n=3)$ and sh-NC group $(n=3)$. The change of tumor size in nude mice was measured every seven days
A

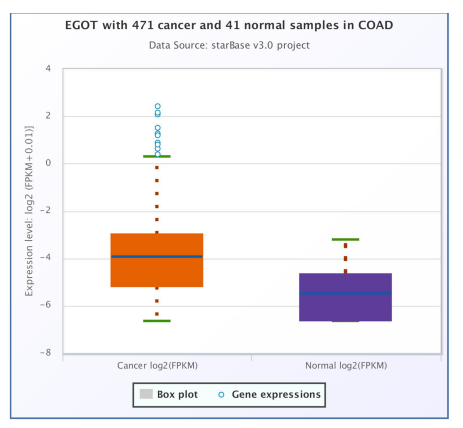

D

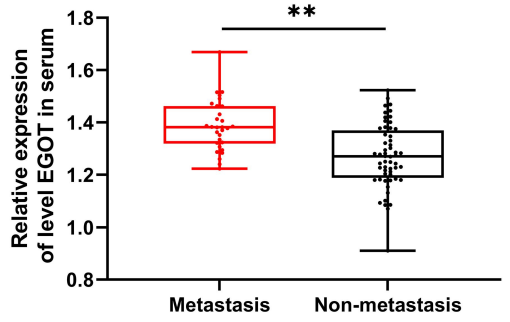

F

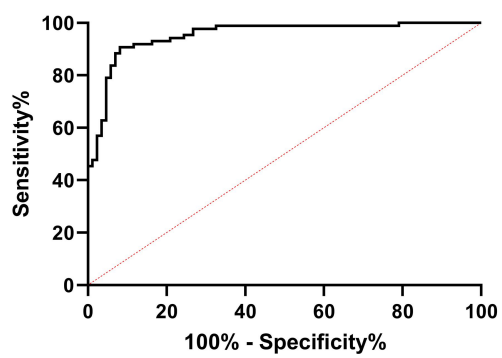

B

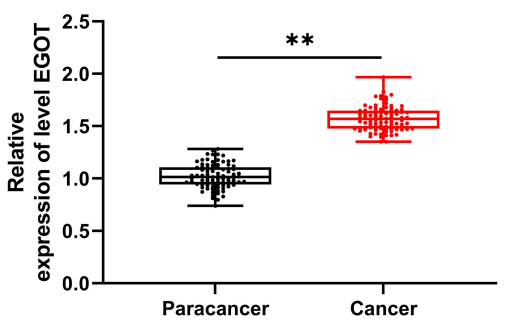

E

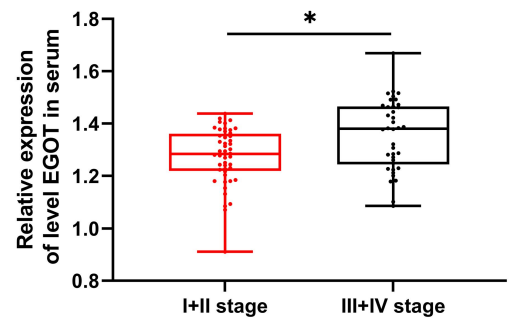

G

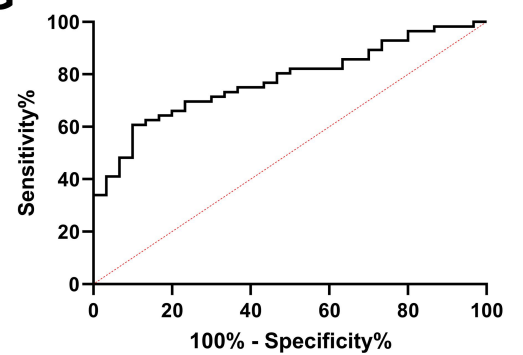

C

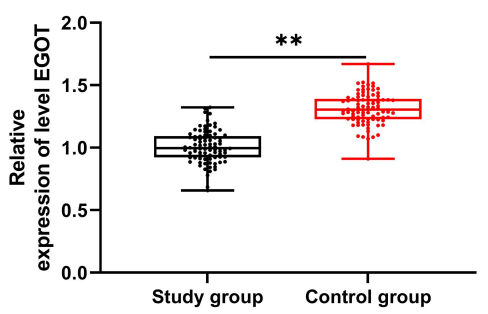

H

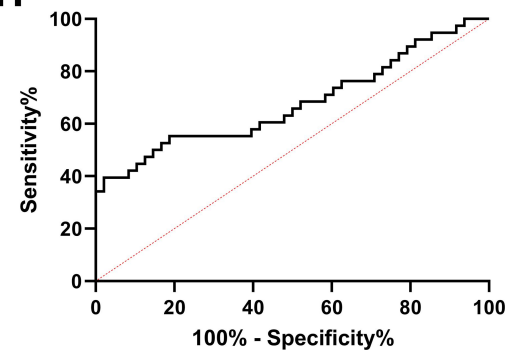

Figure I Expression and diagnostic value of EGOT in CC patients. (A) The starBase online website was used to analyze the EGOT relative expression of CC in TCGA database. (B, C) qRT-PCR was used to detect the relative expression of EGOT in tumor tissue and serum of CC patients. (D, E) qRT-PCR was used to detect the relative expression of EGOT in serum of CC patients with metastasis and nonmetastasis, low stage and high stage of TNM. (F) ROC curve was used to analyze the area under the curve of EGOT in diagnosing CC patients and normal population (0.952), and the best specificity and sensitivity were $91.8 \%$ and $90.6 \%$ when the Youden index was 82.5 . (G) ROC curve was used to analyze the area under the curve of EGOT in diagnosing lymph node metastasis of CC patients (0.777), and the best specificity and sensitivity were $90.0 \%$ and $60.7 \%$ when the Youden index was 50.7 . (H) ROC curve was used to analyze the area under the curve of EGOT in diagnosing low stage and high stage of TNM of CC patients (0.678), and the best specificity and sensitivity were $97.9 \%$ and $37.4 \%$ when the Youden index was 37.3 . $* P<0.01$, $* * P<0.00 I$. 
Table I Relationship Between EGOT and Clinical Data of CC Patients

\begin{tabular}{|c|c|c|c|}
\hline \multirow[t]{2}{*}{ Factors } & \multicolumn{2}{|l|}{ EGOT } & \multirow[t]{2}{*}{$P$-value } \\
\hline & $\begin{array}{l}\text { High } \\
\text { Expression } \\
(n=43)\end{array}$ & $\begin{array}{l}\text { Low } \\
\text { Expression } \\
(n=43)\end{array}$ & \\
\hline Gender & & & 0.822 \\
\hline Male $(n=55)$ & 28 & 27 & \\
\hline Female $(n=3 \mid)$ & 15 & 16 & \\
\hline Age & & & 0.388 \\
\hline$\geq 60$ years old $(n=44)$ & 20 & 24 & \\
\hline$<60$ years old $(n=42)$ & 23 & 19 & \\
\hline Tumor size & & & 0.193 \\
\hline$\geq 3 \mathrm{~cm}(\mathrm{n}=38)$ & 22 & 16 & \\
\hline$<3 \mathrm{~cm}(\mathrm{n}=48)$ & 21 & 27 & \\
\hline Lymphatic metastasis & & & 0.002 \\
\hline Metastasis $(n=30)$ & 22 & 8 & \\
\hline Nonmetastasis $(n=56)$ & 21 & 35 & \\
\hline TNM staging & & & 0.009 \\
\hline$|+| I(n=48)$ & 18 & 30 & \\
\hline III+IV (n=38) & 25 & 13 & \\
\hline KRAS status & & & 0.366 \\
\hline Wild type (n=73) & 35 & 38 & \\
\hline Mutant $(n=13)$ & 8 & 5 & \\
\hline Microsatellite status & & & 0.268 \\
\hline MSS $(n=70)$ & 33 & 37 & \\
\hline$M S I(n=16)$ & 10 & 6 & \\
\hline Cancer location & & & 0.664 \\
\hline Right $(n=38)$ & 18 & 20 & \\
\hline Left $(n=48)$ & 25 & 23 & \\
\hline
\end{tabular}

Abbreviation: $\mathrm{CC}$, colon cancer.

by caliper (volume calculation formula: volume $=(0.5 \times$ length $\times$ width 2)). After 28 days, the mice were killed and the tumor tissues of nude mice were collected.

\section{Statistical Analysis}

GraphPad prism 8 (GraphPad Software Inc., San Diego, California, USA) and SPSS 20.0 (IBM Corporation, Armonk, NY, USA) were used for statistic analysis of all data. Independent-samples $t$-test was applied for comparison between groups. The counting data were represented by percentage (\%) and compared by chi-squared test. One-way ANOVA was used for comparison among groups (represented by F), and LSD $t$-test was applied for posterior comparison. The expression profiles at different time points were verified by repeated measurement analysis of variance $(F)$, and the post-test was completed by Bonferroni. Pearson's test was applied to analyze the connection between genes. The relationship between EGOT and five-year survival of patients with $\mathrm{CC}$ was analyzed by Kaplan-Meier test. The independent prognostic factors of patients with $\mathrm{CC}$ were analyzed by Cox regression. The diagnostic value EGOT in $\mathrm{CC}$ was analyzed by receiver operating curve (ROC). $P<0.05$ was considered significant.

\section{Results \\ EGOT Was Highly Expressed in CC and Had Diagnostic Value}

We first analyzed the relative expression of EGOT in $\mathrm{CC}$ in TCGA database according to starBase online. The results revealed that the EGOT of CC patients' tumor tissues in the database was significantly increased. Then, in order to verify the expression of $E G O T$ in patients with $\mathrm{CC}$, we detected the expression of EGOT in tumor tissues and serum of patients with CC by qRT-PCR (Figure 1A). The findings revealed that the EGOT in tumor tissues and serum of patients with $\mathrm{CC}$ was significantly increased (Figure 1B and C), which was consistent with the database results. This showed that EGOT might be involved in the development of CC. In order to further determine the relationship between EGOT and patients with $\mathrm{CC}$, the patients were separated into high expression group $(n=43)$ and low expression group $(n=43)$ in accordance with the median value of EGOT. By observing the clinical baseline data of patients in both groups (Table 1), it was concluded that the patients in EGOT high expression group had high TNM stage (III+IV), and the probability of lymphatic metastasis increased obviously. Furthermore, we found that $E G O T$ had better diagnostic value in diagnosing patients with $\mathrm{CC}$ (Figure 1D and E), and distinguishing $\mathrm{CC}$ low stage (I+IV) and patients with lymph node metastasis (Figure $1 \mathrm{~F}-\mathrm{H}$ ). Therefore, EGOT was expected to be a potential biological index for diagnosis of $\mathrm{CC}$.

\section{The Five-year Survival Rate of Patients with High EGOT Expression Decreased}

In the above research, we revealed the diagnosis value of $E G O T$ in $\mathrm{CC}$, and we also analyzed the five-year survival of EGOT in patients with CC. Through analysis, we concluded that the five-year survival rate of patients decreased obviously in EGOT high expression group (Figure 2A). In 
A

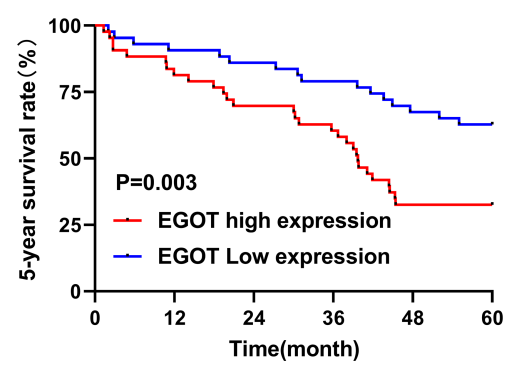

B

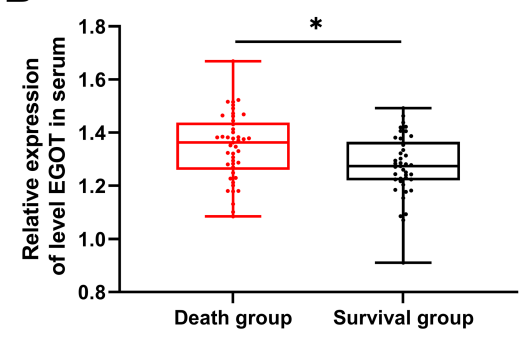

C

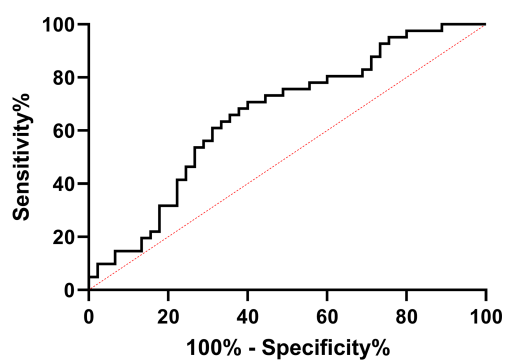

Figure 2 Survival analysis of EGOT and CC patients. (A) K-M survival analysis was used to analyze the five-year survival of patients in the EGOT high and low expression groups. (B) qRT-PCR was used to detect the relative expression of EGOT in serum of dead patients and surviving patients. (C) ROC curve was used to analyze the area under the curve of EGOT in predicting survival and death of CC patients (0.659), and the best specificity and sensitivity were $60.0 \%$ and $70.7 \%$ when the Youden index was 30.7 . $* P<0.0$ I.

addition, Cox regression analysis showed that EGOT and TNM stages were independent prognostic factors of patients with CC (Table 2). Finally, we divided the patients into survival group and death group according to their survival situation. Further comparison showed that the expression of EGOT in the death group was obviously higher than that in survival group, and ROC curve analysis showed that $E G O T$ had a certain value in predicting death of patients with CC (Figure 2B and C).

\section{Upregulation of EGOT Inhibited Autophagy of CC Cells and Promoted Cell Growth}

In the above research, we have revealed the clinical significance of $E G O T$ in $\mathrm{CC}$, but it is not clear whether $E G O T$ is bound up with the development and progression of $\mathrm{CC}$. Therefore, we first detected the $E G O T$ relative expression in CC cells. qRT-PCR detection showed that the $E G O T$ in CC cell strains was significantly increased. Then, we established pcDNA-EGOT, which was transfected into $\mathrm{CC}$ cells. The findings revealed that the relative expression level of EGOT in $\mathrm{CC}$ cells was significantly increased (Figure $3 \mathrm{~A}$ and $\mathrm{B}$ ).
The biological function of the cell was further analyzed. CCK-8 experiment showed that the proliferation activity of CC cells transfected with pcDNA-EGOT was significantly enhanced compared with that of pcDNA-NC (Figure 3C). Transwell experiment showed that after pcDNA-EGOT transfection, the number of cells passing through the membrane was significantly higher than that of CC cells transfected with pcDNA-NC (Figure 3D). In addition, flow cytometry experiment showed that the apoptosis rate of $\mathrm{CC}$ cells transfected with pcDNA-EGOT was lower than that of cells transfected with pcDNA-NC (Figure 3E). Moreover, WB detection showed that the expression of cle-Caspase-3 and Bax protein decreased significantly and the expression of Bcl-2 protein increased (Figure $3 \mathrm{~F}$ ). This indicated that EGOT might be involved in the development of CC. Early studies have shown that EGOT can protect cell growth and development by regulating autophagy. However, it is not clear whether EGOT plays the same role in CC. Therefore, we detected autophagy-related proteins in CC cells transfected with pcDNA-EGOT by WB experiment. The result showed that the relative expressions of LC3II/I and Beclin1

Table 2 Cox Regression Analysis

\begin{tabular}{|c|c|c|c|c|c|c|}
\hline \multirow[t]{2}{*}{ Factors } & \multicolumn{3}{|c|}{ Univariate Cox } & \multicolumn{3}{|c|}{ Multivariate Cox } \\
\hline & Sig. & $\operatorname{Exp}(B)$ & $95 \% \mathrm{Cl}$ & Sig. & $\operatorname{Exp}(B)$ & $95 \% \mathrm{Cl}$ \\
\hline Gender & 0.849 & 1.061 & $0.576-1.954$ & & & \\
\hline Age & 0.430 & 0.789 & $0.439-1.419$ & & & \\
\hline Tumor size & 0.781 & 1.087 & $0.604-1.958$ & & & \\
\hline Lymphatic metastasis & 0.539 & 0.821 & $0.436-1.543$ & & & \\
\hline TNM staging & 0.002 & 0.391 & $0.215-0.708$ & 0.008 & 0.443 & $0.242-0.811$ \\
\hline EGOT & 0.004 & 2.455 & I.328-4.537 & 0.016 & 2.146 & I.150-4.005 \\
\hline KRAS status & 0.321 & 0.691 & $0.332-1.435$ & & & \\
\hline Microsatellite status & 0.219 & 0.643 & $0.318-1.3$ & & & \\
\hline Cancer location & 0.484 & 1.232 & $0.687-2.212$ & & & \\
\hline
\end{tabular}


A

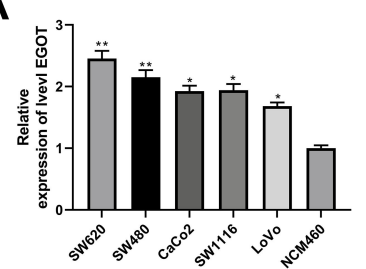

B

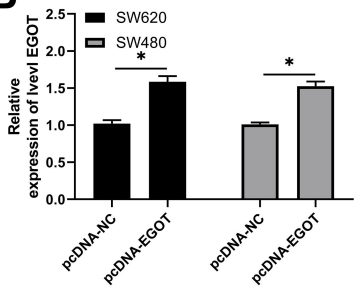

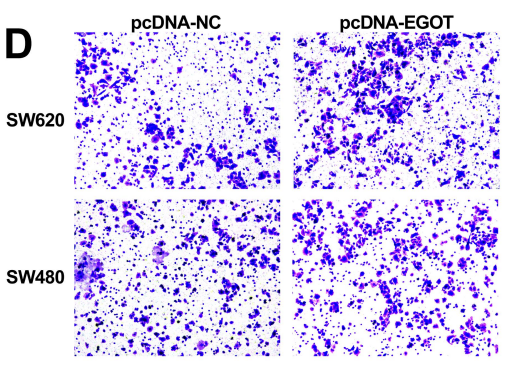

$\mathbf{F}$

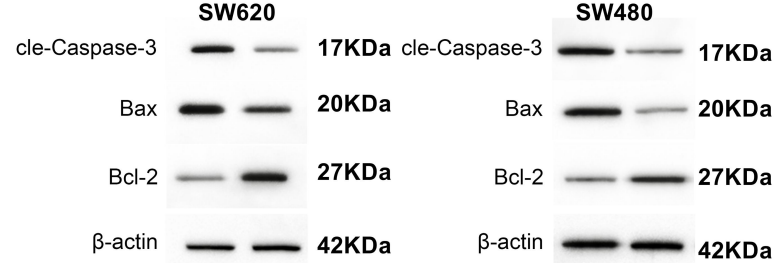

C
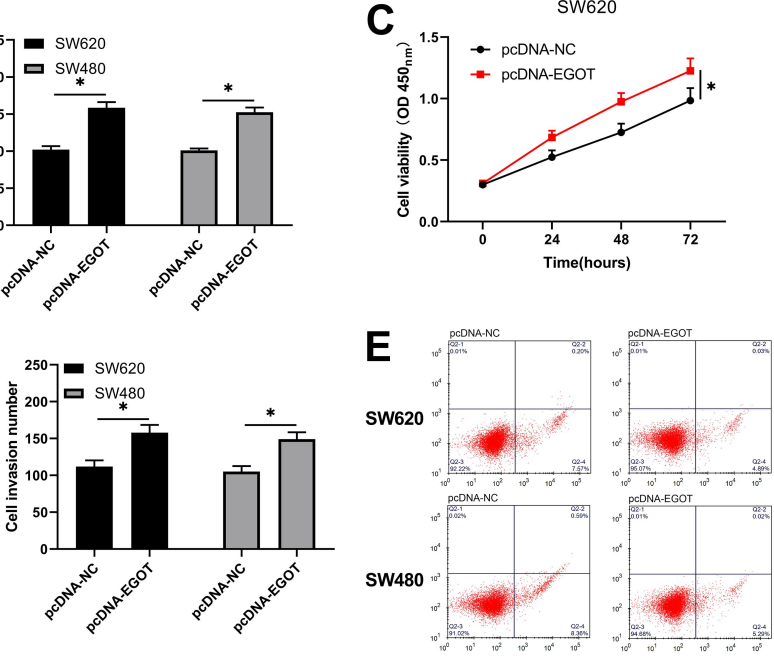

sw620
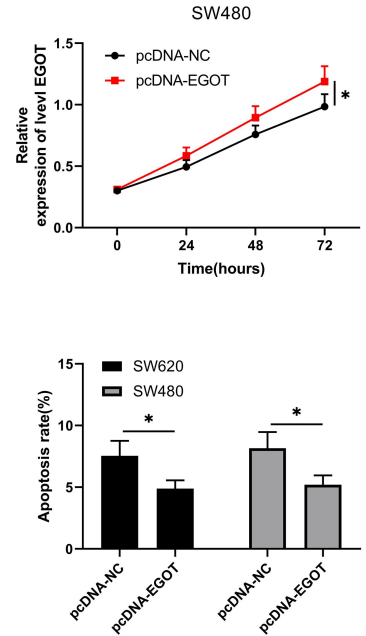
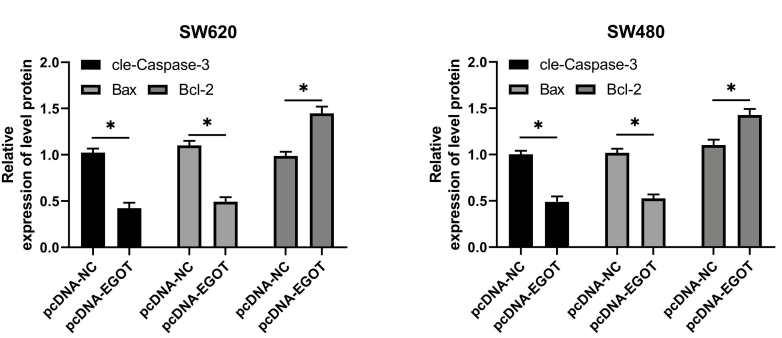

G
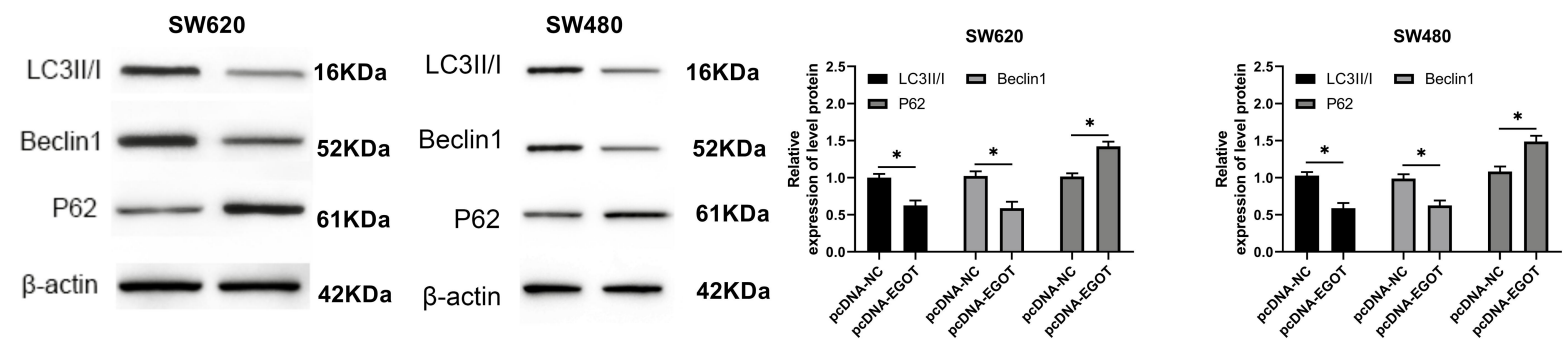

Figure 3 EGOT inhibited autophagy and promoted proliferation and invasion of CC cells. (A) qRT-PCR was used to detect the relative expression of EGOT in CC cells. (B) qRT-PCR was used to detect the relative expression of EGOT in CC cells transfected with pcDNA-EGOT. (C) CCK-8 test was used to detect the change of proliferation ability in CC cells transfected with pcDNA-EGOT. (D) Transwell test was used to detect the change of invasion number in CC cells transfected with pcDNA-EGOT. (E) Flow cytometry was used to detect the apoptosis of CC cells transfected with pcDNA-EGOT. (F, G) WB test was used to detect the changes of relative expression of apoptosisrelated proteins and autophagy-related proteins in CC cells transfected with PcDNA-EGOT. $* P<0.05$.

protein were obviously decreased, while the expression of P62 protein was obviously increased (Figure 3G), In addition, the immunofluorescence staining also showed that the ratio of LC3II/LC3I increased significantly after transfection of pcDNA-EGOT (Figure 4), suggesting that EGOT might facilitate the growth and metastasis of $\mathrm{CC}$ cells by adjusting autophagy.

\section{Knocking Down EGOT Could Suppress the Growth of Tumor Volume in Nude Mice}

In the above study, we revealed the biological function of EGOT in vitro of $\mathrm{CC}$. To further ascertain the value of
EGOT in CC, we established a nude mouse model of allogeneic tumor transplantation. Then the sh-EGOT, which was stably expressed after knocking down, was injected into nude mice for observation. Compared with injection of sh-NC, the tumor volume of nude mice was significantly reduced after injecting CC cells stably transfected with sh-EGOT (Figure 5A and B). By detecting the tumor quality of nude mice, it was also found that the sh$E G O T$ group was lower than the sh-NC group, suggesting that EGOT could suppress tumor growth in nude mice in vivo. In addition, the WB test showed that the expression of cle-Caspase-3, Bax, LC3II/I and Beclin1 in the tumor tissues of nude mice in the sh-EGOT group was 


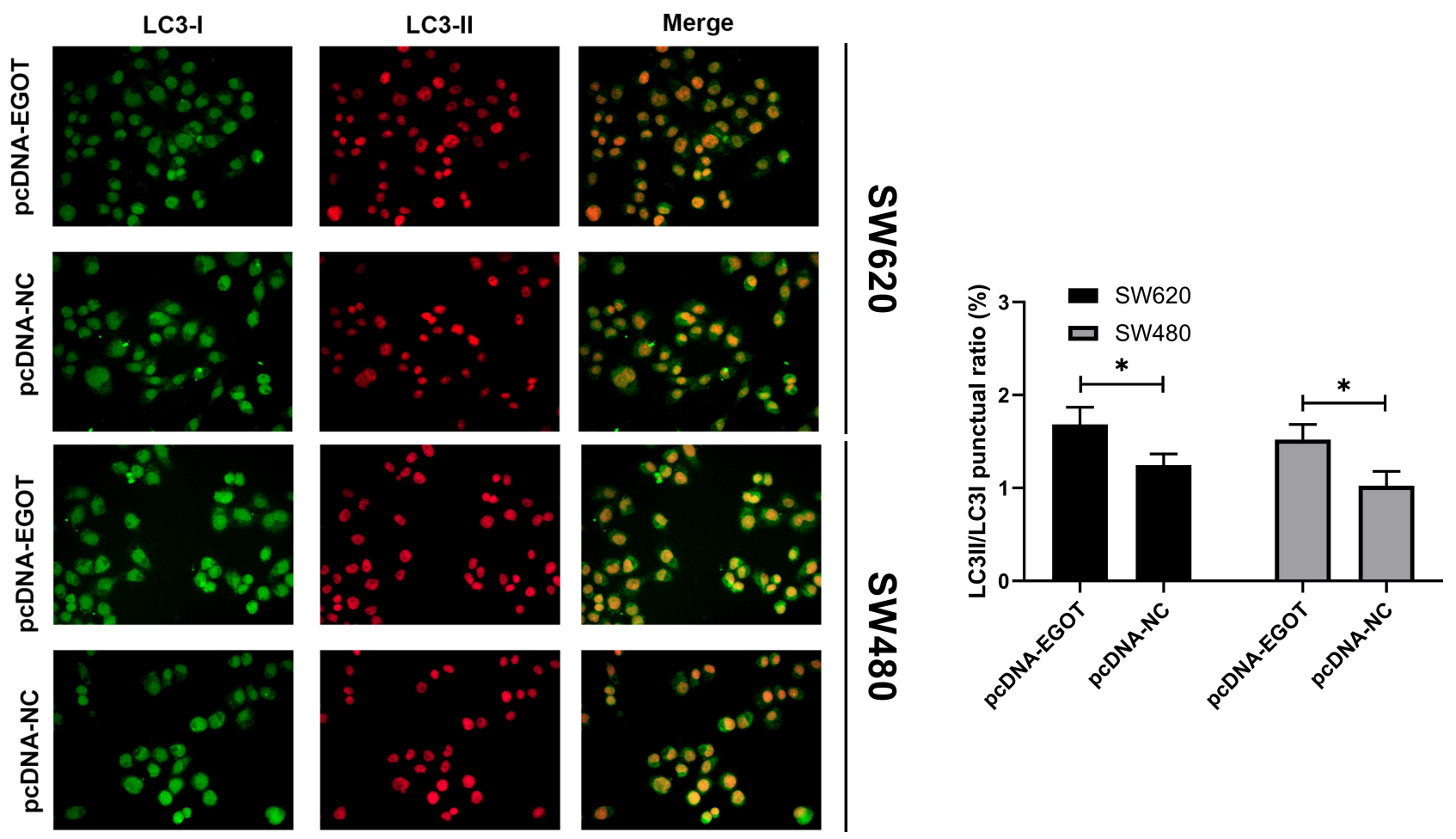

Figure 4 Cell immunofluorescence staining to detect changes in the ratio of LC3II/LC3I in cells after transfection $* P<0.05$. Magnification $\times 200$.

significantly increased, while the expressions of Bcl-2 and P62 protein were declined (Figure 5C). This indicated that EGOT could inhibit the growth and metastasis of CC cells by promoting autophagy. It was expected to become a potential therapeutic target of CC.

\section{Bioinformatics Analysis of EGOT}

Early studies have shown that lncRNA can act as a sponge of microRNA to regulate its downstream target genes and participate in the development and progression of tumors. In this research, in order to ascertain the potential function of EGOT, we predicted the potential microRNA of EGOT, and found that microRNA-33a-5p and microRNA-33b-5p had targeted binding sites with EGOT (Figure 6A). Then we predicted the target genes downstream of microRNA-33a$5 p$ and microRNA-33b-5p and drew the ceRNA network map (Figure 6B). In addition, nine potential GO functions and 10 potential signal pathways were found by GO enrichment and KEGG analysis (Figure 6C and D), which is the potential direction for us to explore $E G O T$ in the future.

\section{Discussion}

Although some progress has been made in the treatment of $\mathrm{CC}$, clinicians are still faced with the challenge of how to improve the early diagnosis of diseases by developing methods that can detect malignant tumors. ${ }^{23}$ In this research, we found that EGOT was expressed to a high degree in cases of $\mathrm{CC}$, and it had high clinical value in diagnosing patients with $\mathrm{CC}$, lymphatic metastasis and TNM staging. In addition, we also found that EGOT could inhibit the growth and metastasis of CC cells by regulating autophagy, which was expected to be a potential target and diagnostic index for treatment of CC.

LncRNA is a long-chain noncoding RNA. At present, many studies have shown that IncRNA has high clinical value in the diagnosis of tumor. ${ }^{24-26}$ For example, the research of Liu et $\mathrm{al}^{27}$ have shown that LncRNA H19 can be used as a cancer biomarker with high value in the detection and diagnosis of human cancer. EGOT, as a newly discovered lncRNA, is found to be highly expressed in glioma and other diseases in early studies. ${ }^{28}$ Furthermore, it is found that EGOT can promote the apoptosis of $\mathrm{CC}$ through microRNA-33b-5p/CROT axis. ${ }^{29}$ However, the predictive value of $E G O T$ in clinical diagnosis of $\mathrm{CC}$ is still unclear. In this research, we first analyzed the expression of EGOT in CC. qRT-PCR analysis revealed that the relative expression of EGOT was high in serum, tumor tissues and CC cells of patients with $\mathrm{CC}$, which was consistent with the expression in TCGA database. It was also concluded that EGOT was bound up with TNM staging and lymphatic metastasis. 
A

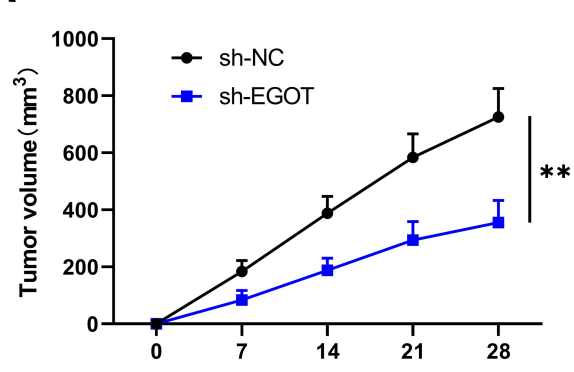

C

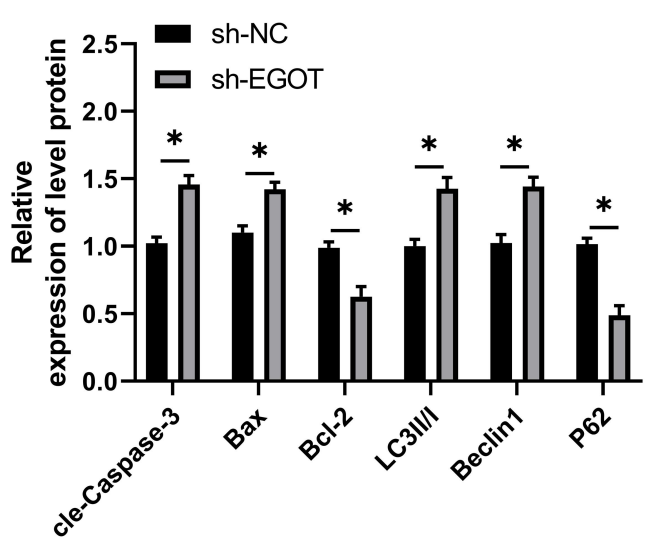

B
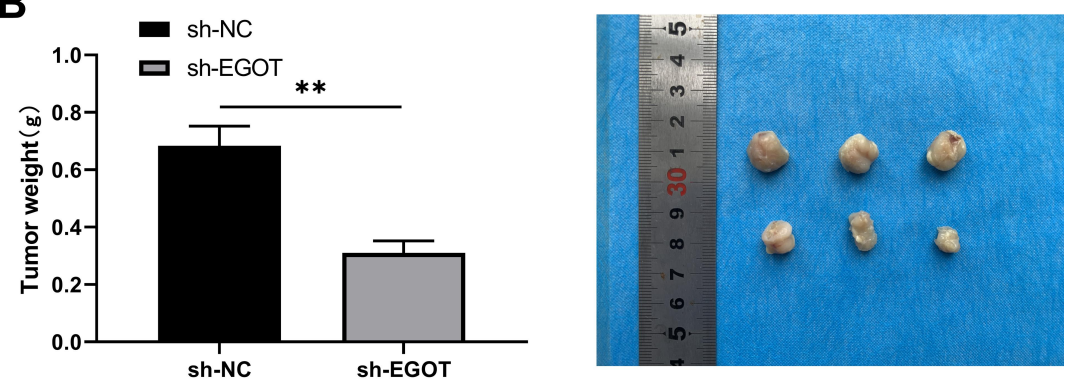

cle-Caspase-3

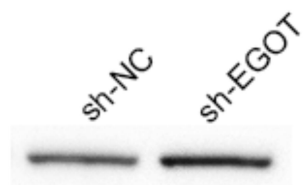

Bax

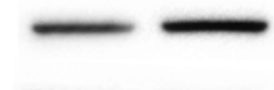

$\mathrm{Bcl}-2$

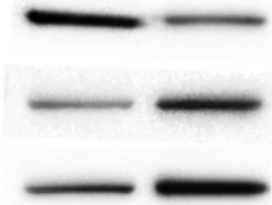

Beclin1

P62

$\beta$-antin
$17 \mathrm{KDa}$

20KDa

27KDa

16KDa

$52 \mathrm{KDa}$

$61 \mathrm{KDa}$

42KDa

Figure 5 EGOT could inhibit growth of tumor volume in nude mice. (A) Changes of tumor volume in nude mice within 28 days. (B) Changes of tumor quality in nude mice after death for 28 days. (C) WB was used to detect the changes of apoptosis-related proteins and autophagy-related proteins in tumor tissues of nude mice. $* P<0.05$, $* * P<0.01$.

Serum detection is a widely used diagnostic standard in diagnosing tumors at present. ${ }^{30}$ Early research has also revealed that detecting the lncRNA in serum can be used as a potential index for diagnosis of CC. ${ }^{31}$ To determine the diagnostic value of $E G O T$ in $\mathrm{CC}$, we drew a ROC curve. The results showed that EGOT had certain diagnostic value in diagnosing patients with $\mathrm{CC}$, lymphatic metastasis and TNM staging, especially in diagnosing tumor and lung tumor patients, and the area under the curve was greater than 0.9 , which was a better clinical potential diagnostic index. In addition, we also analyzed the relationship between EGOT and five-year survival of patients with CC. Through the analysis, we found that EGOT and TNM staging were independent factors for the prognosis of patients with $\mathrm{CC}$. We also found that EGOT had a certain value in predicting five-year survival of $\mathrm{CC}$ patients with an area under the ROC curve greater than 0.6 , but it was still not ideal.
Studies have shown that EGOT has the ability to regulate autophagy, and facilitates the apoptosis of tumor cells by adjusting autophagy. ${ }^{32}$ However, whether EGOT has the same effect in $\mathrm{CC}$ has not been confirmed by relevant studies. Therefore, we established the overexpression vector of pcDNA-EGOT to explore the value of $E G O T$ in CC. Through experiments, we concluded that transfection of pcDNA-EGOT promoted the growth and invasiveness of CC cells and inhibited apoptosis. This showed that EGOT participated in the development of CC. The ratio of LC3-II/I is an important marker of autophagy. P62 is also considered as a selective autophagy substrate. More and more evidences show that p62 can act as a receptor for selective autophagy products of various ubiquitinated substrates. ${ }^{33}$ In WB experiment, we found that the expressions of cle-Caspase-3, Bax, LC3II/I and Beclin1 were obviously decreased, and the expressions of p62 and Bcl-2 were increased in cells transfected with pcDNA-EGOT, which indicated that EGOT promoted the 
A

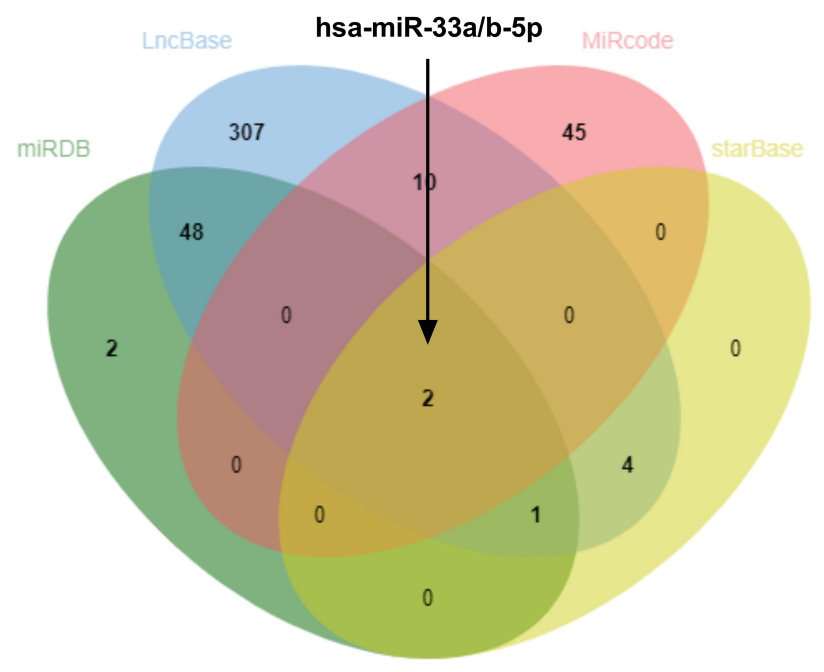

C

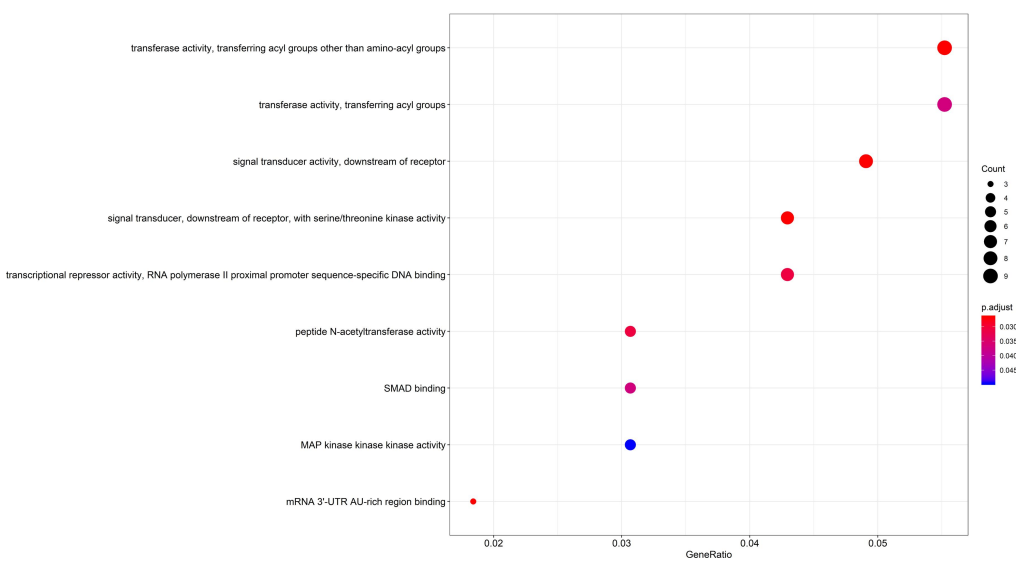

B

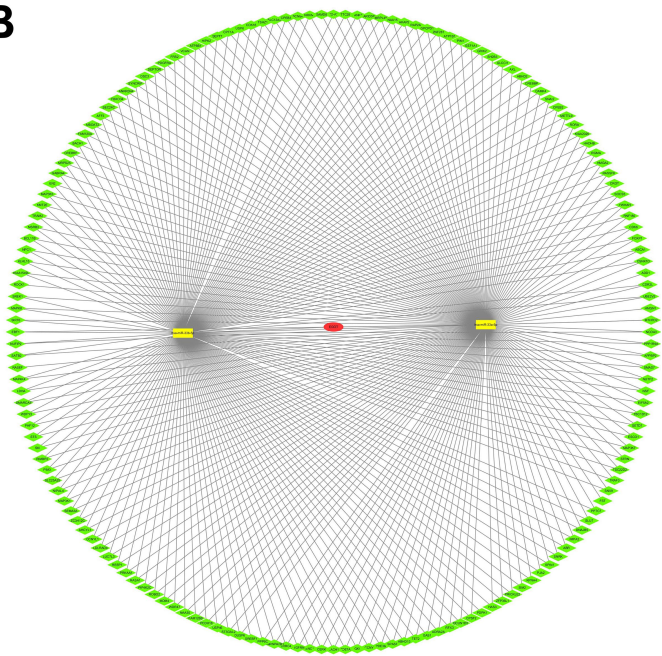

D

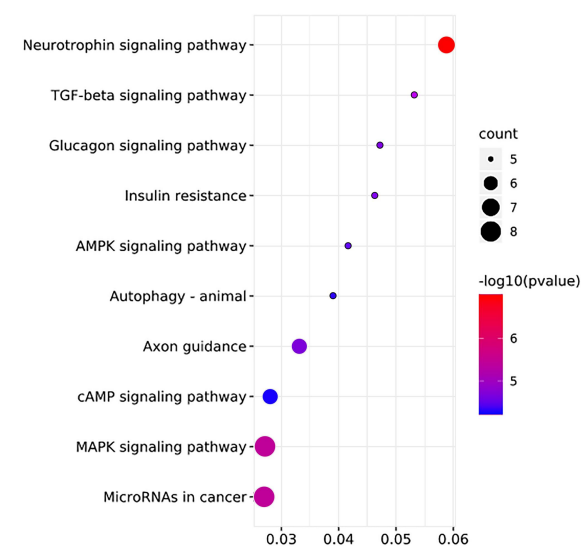

Figure 6 EGOT function analysis. (A) Common potential microRNA of EGOT predicted by several online sites. (B), EGOT-microRNA-mRNA network map drawn by Cytoscape (red was IncRNA, yellow was microRNA, and green was mRNA). (C) Analysis of potential GO function of target gene. (D) Analysis of potential KEGG signaling pathway of target gene.

development of $\mathrm{CC}$ by inhibiting autophagy. In order to further verify our conclusion, we also carried out in vivo experiments. The findings showed that the tumor size and mass of nude mice were significantly decreased after knocking down EGOT, and the relative expression of autophagy protein and apoptosis protein in tumor tissue was contrary to that in vitro, which verified that EGOT could regulate $\mathrm{CC}$.

At the end of the study, we predicted the potential microRNA-mRNA of EGOT to further seek the potential mechanisms of lncRNA. Early studies have shown that lncRNA can be used as a microRNA sponge to regulate downstream target genes and participate in tumorigenesis. Through prediction, we found that microRNA-33a-5p and microRNA-33b-5p had targeted binding sites with EGOT, Then we predicted
microRNA-33a-5p and microRNA-33b-5p downstream target genes and conducted enrichment analysis, and found that there was a connection between EGOT and autophagy signaling pathway. However, the specific relationship has not been proved, which is also our main research direction in the future.

Compared with the study of $\mathrm{Ni}$ et al, ${ }^{29}$ this study was designed to analyze the diagnostic value of LncRNA $E G O T$ in $\mathrm{CC}$, and the ceRNA map was mapped by miR and its downstream mRNA which could bind to LncRNA EGOT. In addition, we also carried out a nude mouse experiment to verify the inhibitory effect of LncRNA EGOT on tumor growth in vivo. However, there are some deficiencies in this research. First, it is not clear whether EGOT can mediate microRNA to regulate autophagy of CC. Second, autophagy inhibitors are not used to 
intervene in cells or nude mice in this study. Therefore, we hope to carry out more basic research in the future to improve our research conclusions.

\section{Conclusion}

To sum up, EGOT is highly expressed in $\mathrm{CC}$ and has high diagnostic value. In addition, inhibition of EGOT can promote autophagy of CC cells and inhibit cell growth and metastasis, which is expected to become a potential therapeutic index.

\section{Acknowledgments}

The authors received no funding for this work.

\section{Disclosure}

The authors report no conflicts of interest in this work.

\section{References}

1. Orangio GR. The Economics of Colon Cancer. Surg Oncol Clin N Am. Apr 2018;27(2):327-347. doi:10.1016/j.soc.2017.11.007

2. Bray F, Ferlay J, Soerjomataram I, Siegel RL, Torre LA, Jemal A. Global cancer statistics 2018: GLOBOCAN estimates of incidence and mortality worldwide for 36 cancers in 185 countries. $C A$ Cancer J Clin. 2018;68(6):394-424. doi:10.3322/caac.21492

3. Weinberg BA, Marshall JL. Colon cancer in young adults: trends and their implications. Curr Oncol Rep. 2019;21(1):3. doi:10.1007/ s11912-019-0756-8

4. Zhou R, Zhang J, Zeng $\mathrm{D}$, et al. Immune cell infiltration as a biomarker for the diagnosis and prognosis of stage I-III colon cancer. Cancer Immunol Immunother. 2019;68(3):433-442. doi:10. 1007/s00262-018-2289-7

5. Zhang X, Zhang H, Shen B, Sun XF. Chromogranin-A expression as a novel biomarker for early diagnosis of colon cancer patients. Int J Mol Sci. 2019;20(12).

6. Attallah AM, El-Far M, Ibrahim AR, et al. Clinical value of a diagnostic score for colon cancer based on serum CEA, CA19-9, cytokeratin-1 and mucin-1. Br J Biomed Sci. 2018;75(3):122-127. doi:10.1080/09674845.2018.1456309

7. Wang J, Zhu S, Meng N, He Y, Lu R, Yan GR. ncRNA-encoded peptides or proteins and cancer. Mol Ther. 2019;27(10):1718-1725. doi:10.1016/j.ymthe.2019.09.001

8. Xu J, Bai J, Xiao J. Computationally modeling ncRNA-ncRNA crosstalk. Adv Exp Med Biol. 2018;1094:77-86.

9. Zhao Z, Sun W, Guo Z, Zhang J, Yu H, Liu B. Mechanisms of lncRNA/microRNA interactions in angiogenesis. Life Sci. 2020;254:116900. doi:10.1016/j.lfs.2019.116900

10. Ferre F, Colantoni A, Helmer-Citterich M. Revealing protein-lncRNA interaction. Brief Bioinform. 2016;17(1):106-116. doi:10.1093/bib/ bbv031

11. Wu Q, Meng WY, Jie Y, Zhao H. LncRNA MALAT1 induces colon cancer development by regulating miR-129-5p/HMGB1 axis. $J$ Cell Physiol. 2018;233(9):6750-6757. doi:10.1002/jcp.26383

12. Huang JZ, Chen M, Chen D, et al. A peptide encoded by a putative lncRNA HOXB-AS3 suppresses colon cancer growth. Mol Cell. 2017;68(1):171-184 e176. doi:10.1016/j.molcel.2017.09.015

13. Zhou F, Shen F, Zheng Z, Ruan J. The LncRNA XIRP2-AS1 predicts favorable prognosis in colon cancer. Onco Targets Ther 2019;12:5767-5778. doi:10.2147/OTT.S215419
14. Kawasaki Y, Miyamoto M, Oda T, et al. The novel lncRNA CALIC upregulates AXL to promote colon cancer metastasis. EMBO Rep. 2019;20(8):e47052. doi:10.15252/embr.201847052

15. Peng W, Wu J, Fan H, Lu J, Feng J. LncRNA EGOT promotes tumorigenesis via hedgehog pathway in gastric cancer. Pathol Oncol Res. 2019;25(3):883-887. doi:10.1007/s12253-017-0367-3

16. Wu S, Ai H, Zhang K, Yun H, Xie F. Long non-coding RNA EGOT promotes the malignant phenotypes of hepatocellular carcinoma cells and increases the expression of HMGA2 via down-regulating miR-33a-5p. Onco Targets Ther. 2019;12:11623-11635. doi:10.21 47/OTT.S218308

17. Xu SP, Zhang JF, Sui SY, et al. Downregulation of the long noncoding RNA EGOT correlates with malignant status and poor prognosis in breast cancer. Tumour Biol. 2015;36(12):9807-9812. doi:10.1007/ s13277-015-3746-y

18. Jin L, Quan J, Pan X, et al. Identification of lncRNA EGOT as a tumor suppressor in renal cell carcinoma. Mol Med Rep. 2017;16 (5):7072-7079. doi: 10.3892/mmr.2017.7470

19. Li JH, Liu S, Zhou H, Qu LH, Yang JH. starBase v2.0: decoding miRNA-ceRNA, miRNA-ncRNA and protein-RNA interaction networks from large-scale CLIP-Seq data. Nucleic Acids Res. 2014;42 (D1):D92-97. doi:10.1093/nar/gkt1248

20. Issue information-declaration of Helsinki. J Bone Miner Res. 2018;33 (12):BMi-BM ii.

21. Livak KJ, Schmittgen TD. Analysis of relative gene expression data using real-time quantitative PCR and the 2(-Delta Delta C(T)) method. Methods. 2001;25(4):402-408. doi:10.1006/meth.2001.1262

22. MacArthur Clark JA, Sun D. Guidelines for the ethical review of laboratory animal welfare People's Republic of China National Standard GB/T 35892-2018 [Issued 6 February 2018 Effective from 1 September 2018]. Animal Model Exp Med. 2020;3(1):103-113. doi:10.1002/ame2.12111

23. Arnold MW. Colon cancer: the road traveled. Surg Oncol Clin N Am. 2018;27(2):xv-xviii. doi:10.1016/j.soc.2017.11.012

24. Chao Y, Zhou D. IncRNA-D16366 is a potential biomarker for diagnosis and prognosis of hepatocellular carcinoma. Med Sci Monit. 2019;25:6581-6586. doi:10.12659/MSM.915100

25. Yang J, Li C, Mudd A, Gu X. LncRNA PVT1 predicts prognosis and regulates tumor growth in prostate cancer. Biosci Biotechnol Biochem. 2017;81(12):2301-2306. doi:10.1080/09168451.2017.1387048

26. Loewen G, Jayawickramarajah J, Zhuo Y, Shan B. Functions of lncRNA HOTAIR in lung cancer. $J$ Hematol Oncol. 2014;7(1):90. doi:10.1186/s13045-014-0090-4

27. Liu C, Chen L, You Z, et al. Association between IncRNA H19 polymorphisms and cancer susceptibility based on a meta-analysis from 25 studies. Gene. 2020;729:144317. doi:10.1016/j.gene.2019.144317

28. Wu Y, Liang S, Xu B, et al. Long noncoding RNA eosinophil granule ontogeny transcript inhibits cell proliferation and migration and promotes cell apoptosis in human glioma. Exp Ther Med. 2017;14 (4):3817-3823. doi:10.3892/etm.2017.4949

29. Ni Y, Li C, Bo C, et al. LncRNA EGOT regulates the proliferation and apoptosis of colorectal cancer by miR-33b-5p/CROT axis. Biosci Rep. 2020. doi:10.1042/BSR20193893

30. Slaby O. Non-coding RNAs as biomarkers for colorectal cancer screening and early detection. Adv Exp Med Biol. 2016;937:153-170.

31. Wang J, Li H, Zhang C, Xue L, Cai Z. Cervical carcinoma high-expressed long non-coding RNA 1 may promote growth of colon adenocarcinoma through interleukin-17A. Oncol Lett. 2019;18(2):1491-1496.

32. Xu S, Wang P, Zhang J, et al. Ai-lncRNA EGOT enhancing autophagy sensitizes paclitaxel cytotoxicity via upregulation of ITPR1 expression by RNA-RNA and RNA-protein interactions in human cancer. Mol Cancer. 2019;18(1):89.

33. Loos F, Xie W, Sica V, et al. Artificial tethering of LC3 or p62 to organelles is not sufficient to trigger autophagy. Cell Death Dis. 2019;10(10):771. doi:10.1038/s41419-019-2011-5 


\section{Publish your work in this journal}

Cancer Management and Research is an international, peer-reviewed open access journal focusing on cancer research and the optimal use of preventative and integrated treatment interventions to achieve improved outcomes, enhanced survival and quality of life for the cancer patient.
The manuscript management system is completely online and includes a very quick and fair peer-review system, which is all easy to use. Visit http://www.dovepress.com/testimonials.php to read real quotes from published authors. 